\title{
NOTES ET INFORMATIONS
}

Nils Johan Teodor Odhner. - Le Professeur Teodor Odhner, né à Lund (Suède), le 25 février 1879, est mort à 49 ans à Stockholm, le 29 octobre 1928. Il avait consacré une grande part de son activité à l'étude des trématodes. Ses travaux en la matière jouissent d'une autorité qui est due à leur base solidement établie. La classification naturelle de beaucoup de groupes était à peine ébauchée ou même inexistante avant les publications de T. Odhner et il est actuellement impossible d'étudier les trémalodes sans avoir recours journellement à ses ouvrages principaux : Die Trematoden des arktischen Gebietes (1905), Nordostafrikanische Trematoden (1910), Zum natürlichen System der digenen Trematoden (1911-1913).

Esprit critique, mais constructeur, T. Odhner a toujours travaillé avec beaucoup de méthode, non pas en systématicien, mais en morphologiste et faunisticien.

Deux expéditions scientifiques ont fait appel à sa collaboration : il a pris part à l'expédition du $\mathrm{D}^{r}$ Kolthoff au Spitzberg et dans l'Est du Groënland (1900), ainsi qu'à celle dirigée par L.-A. Jägerskiöld en Egypte, au Soudan et dans la presqu'ile du Sinaï (1900-1901).

T. Odhner estimait peu les honneurs, il fut néanmoins élu vice-secrétaire de l'Académie des Sciences de Suède (1925) et membre de l'Académie des Sciences de Norvège. Il avait été lecteur de la chaire de zoologie à l'Université d'Upsala, puis professeur de zoologie à l'Université de Christiania et finalement intendant du département des invertébrés au Muséum d'Histoire Naturelle de Stockholm.

Le souvenir de T. Odhner restera cher aux parasitologues français.

$$
\text { R.-Ph. Dollfus. }
$$

Helminthologie. - Jusqu'ici, en dehors des traités de parasitologie ou de zoologie médicale, les parasitologues, les médecins et les vétérinaires n'avaient pas à leur disposition de traité d'helminthologie. Pourtant, cette branche de la parasitologie a pris une telle importance aux points de vue théorique et économique qu'un ouvrage exclusivement consacré aux helminthes était vivement désiré.

Cette lacune vient d'être comblée par H.-A. Baylis, le savant helminthologiste du British Museum. Dans un livre d'un peu plus de 300 pages, illustré de 200 figures, intitulé A Manual of helminthology medical and veterinary, on trouvera condensées toutes les notions essentielles de

AnNales de Parasitologie, $\mathrm{T}$, VII, $\mathrm{x}^{\circ} 3,-1^{\text {er }}$ mai $1929, \mathrm{p} .247-248$. 
l'helminthologie. Cet ouvrage, publié par Baillière, Tindall et Cox, fait partie d'une série parasitologique où ont déjà paru les deux magnifiques volumes du Traité de protozoologie de C.-M. Wenyon et où d'autres ouvrages de parasitologie sont annoncés.

Le domaine de l'étude des vers parasites est si vaste que l'auteur a dû se limiter aux helminthes de l'homme et des animaux domestiques et encore en ne comprenant parmi ces derniers que les espèces les plus communes. Les descriptions ne renferment que les détails strictement indispensables pour la détermination des helminthes expulsés pendant la vie ou découverts au cours d'autopsies, mais l'avantage d'un ouvrage de ce genre est de rassembler des documents épars dans la littérature scientifique et souvent très difficiles à se procurer. Toutefois l'auteur a dù renoncer à donner la synonymie développée des genres et des espèces et leur histoire clinique complète. Une heureuse idée a été de présenter sous forme de tableaux les caractères des espèces, pour les genres où elles sont nombreuses. Cette disposition est bien préférable à l'emploi des clefs dichotomiques, avec lesquelles il est si facile de s'égarer.

Dans son exposé, l'auteur a délibérément adopté l'ordre naturel de la classification, d'après les connaissances actuelles sur la phylogénie des divers types. Un index suflit pour donner la classification par hôtes. Baylis pense qu'à côté des quelques parasites connus chez l'homme et les animaux domestiques de son entourage, il y a probablement des centaines d'autres espèces vivant chez les animaux sauvages; il a donc tenu compte de ce fait dans l'exposé systématique des divers groupes, de sorte que le lecteur est mis au courant des vues d'ensemble de la classification.

Ecrit par un spécialiste particulièrement qualifié, ce Traité fera connaitre l'état aetuel de la nomenclature helminthologique et de la biologie des helminthes et facilitera ainsi les investigations des chercheurs.

M. LANGeron.

Culture des trypanosomes, et en particulier T. inopinatum, en milieu liquide sucré. - On sait que Ponselle (Ann. de Parasit., I, 1923, p. 155) a cultivé pour la première fois Trypanosoma inopinatum Ed. et Et. Sergent sur un milieu composé de sang de lapin défibriné et d'eau bidistillée à partíes égales, mélange réalisant les conditions indispensables au développement de ces flagellés, à savoir l'hypotonicité et l'acidité. Il est facile de constater en effet combien $T$. inopinatum est sensible aux plus légères modifications de ces facteurs, et que l'acidité en particulier ne peut varier que dans des limites très étroites $(p \mathrm{H}$ optimum 5,5). De plus, la moindre trace d'un sel quelconque, ne serait-ce que le fait d'utiliser l'eau ordinaire, même acidifiée, en place d'eau bidistillée, inhibe tout développement.

Cependant, par addition de certaines substances au liquide de culture, nous avons pu arriver à réduire l'importance de ces facteurs. C'est ainsi 
qu'en utilisant un milieu composé d'eau distillée, glycosée à 2 pour 100 , et mélangée à parties égales avec du sang de lapin défibriné, puis décomplémenté pendant 1 heure à $56^{\circ}$, nous avons obtenu des cultures extrèmement abondantes de $T$. inopinatum. On peut alors faire varier le $p \mathrm{H}$ sans inconvénient de 5,5 à 7,8 , et l'on ne modifie pas les résultats en utilisant l'eau ordinaire qui convient mieux encore que l'eau distillée dans ce cas. Nous avons même constaté, ce qui paraît assez paradoxal, que la présence de glycose permet d'introduire dans le milieu jusqu’à 1 gr. 5 pour 1.000 de chlorure de sodium sans gêner le développement des trypanosomes.

Ce milieu permet en outre une très longue conservation des cultures : elles sont encore très abondantes au bout de trois mois, et il persiste encore de nombreuses formes au bout de quatre mois et demi, alors qu'en milieu Ponselle ordinaire, à $15^{\circ}$, la culture ne persiste guère après un mois et demi, à moins de conserver les tubes à la glacière. Nous avons utilisé le glycose de préférence à la peptone qui donne des résultats beaucoup moins constants, sauf dans un cas où nous avons obtenu une culture persistant deux mois et demi.

Ce milieu liquide sucré semble même mieux convenir que le milieu de Row pour les trypanosomes autres que $T$. inopinatum. En particulier, une souche pure mixte de $T$. parroti et $T$. sergenti Brumpt, 1923, isolée en culture par le mème auteur en 1928, donne sur ce milieu des cultures exubérantes. Pour T. cruzi et $T$. lewisi, le développement est beaucoup plus long; les cultures sont moins abondantes, mais leur longévité est assez importante : deux mois et demi à quatre mois, temps au bout duquel on peut les réensemencer avec succès sur le même milieu ou sur des milieux ordinaires. Ici encore, comme pour $T$. inopinatum, la question du $\mathrm{pH}$ intervient relativement peu et on peut utiliser indifféremment l'eau ordinaire ou l'eau bidistillée.

H. GaLliakd.

\section{Erratum}

C'est par suite d'un oubli regrettable que nous avons omis de mentionner, dans un article récemment paru (Ann. de Parasitologie, VII, 1929, p. 57), l’origine des souches de spirochètes utilisées dans nos expériences. La souche de Treponema crociduræ Léger, 1917, fut apportée de Dakar par le $\mathrm{D}^{\mathrm{r}}$ Mathis qui l'y avait isolée. C'est actuellement la seule qui existe en Europe. La souche de $T$. hispanicum fut envoyée par le $D^{r}$ S. de Buen qui découvrit ce parasite en 1926.

Nous exprimons à MM. Mathis et de Buen tous nos regrets pour cet oubli bien involontaire.

H. Galliakd. 